Territorialities and inequalities of orientation: what the terrain does to these two concepts and their interrelations. (Reflections from the case of France)

\title{
Pierre Champollion
}

Docteur (PhD) habilité à diriger des recherches - Sciences de l'éducation et de la formation. EA 4571 ECP Lyon2 UJM (chercheurtitulaire) / ESO - Caen (chercheurassocié) / Contributeur CNESCO. Président de

"Observatoireéducation et territoires"

E-mail: pierre.champollion@univ-grenoble-alpes.fr

Traducción: Dra. Matilde Balduzzi

E-mail: matzi09@yahoo.com.ar

\section{Resumen}

Salvo en los últimos años -e incluso muy raramentelas relaciones entre territorialidades, o territorios simbólicos y desigualdades específicas de orientación han sido escasamente estudiadas. La literatura en la materia, en lengua francesa, portuguesa y española, concerniente específicamente a esta compleja cuestión reúne hoy solo algunos pocos artículos científicos, a veces generales (Champollion, 2015), a veces vinculados a un contexto territorial particular, como sucede con la zona de montaña (Champollion, 2019). La primera parte de este artículo abordará la diferenciación progresiva de la noción de desigualdad educativa que, actualmente, aparece cada vez más como multifactorial (contextual, escolar en términos de rendimiento, de orientación, etc.). La segunda parte tratará sobre la diferenciación progresiva de las diferentes dimensiones de la noción de territorio -en particular, institucional, vivido, simbólico, interiorizado- que ha visto surgir hacia fines del siglo XX, la territorialidad. La tercera parte, finalmente, una vez trazado este cuadro conceptual, se centrará en el impacto de la territorialidad sobre la orientación escolar y profesional de los alumnos.

Palabras Clave: Desigualdad, orientación, territorio, territorialidad.

\section{Abstract}

Except - and still very rarely - in recent years, the relationships between territorialities, or symbolic territories, and specific inequalities of orientation have hardly been really studied. Francophone, Lusophone and Hispanophone literatures on the subject, dealing specifically with this complex issue, today only brings together a few rare scientific articles, sometimes general (Champollion, 2015), sometimes linked to a particular territorial context, in this case the mountain (Champollion, 2019). The first part of this review article will address the progressive differentiation of the notion of educational inequality which appears today to be more and more multifactorial (contextual, educational in terms of performance, orientation, etc.). The second part, it will deal with the progressive differentiation of the different dimensions of the notion of territory - institutional, lived, symbolic, internalized, in particular - which saw recently, at the end of the XXth century, the emergence of territoriality. The third part, finally, will focus more particularly, once this conceptual framework has been set, on the impact of territoriality on the academic and professional orientation of students.

Key words: Inequality, orientation, territory, territoriality.

CHAMPOLLION, P. (2020) "Territorialidades y desigualdades de orientación: los espacios ante estos dos conceptos y sus interrelaciones (Reflexiones a partir del caso de Francia)". Espacios en Blanco. Revista de Educación, $\mathrm{N}^{\circ} 30$, vol. 2, jul./dic. 2020, pp. 335-349. Universidad Nacional del Centro de la Provincia de Buenos Aires, Argentina

DOI: https://doi.org/10.37177/UNICEN/EB30-281 


\section{Introducción}

Las relaciones entre territorios -en general- y la educación -en sentido amplio ${ }^{1}$ comienzan a estar hoy relativamente bien documentadas. Los impactos de los territorios son analizados a partir de la densidad, de la distancia o proximidad de la oferta de formación, del transporte escolar, del financiamiento de las colectividades territoriales, etc. Pero las relaciones entre territorialidades, o territorios simbólicos, y desigualdades específicas de orientación, no han sido en cambio, hasta el presente, sino apenas y verdaderamente estudiadas. La literatura en lengua francesa, portuguesa y española en la materia, que trata específicamente sobre esta cuestión compleja, reúne hoy solo algunos pocos artículos científicos, a veces generales (Champollion, 2015), a veces vinculados a un contexto territorial particular, como sucede con la zona de montaña (Champollion, 2019a).

La primera parte de este artículo abordará rápidamente la cuestión de la diferenciación progresiva de la noción de desigualdad educativa que aparece hoy cada vez más como multifactorial (contextual, escolar en términos de rendimiento, de orientación, de inserción, etc.), centrándose particularmente en las desigualdades de orientación. La segunda parte seguirá la pista de la diferenciación progresiva de las distintas dimensiones del concepto de territorio -institucional, vivido, simbólico (de su parte interiorizada), en particular- que ha visto recientemente, hacia fines del siglo XX, emerger la territorialidad a partir de poner en evidencia el territorio simbólico, el cual será analizado más precisamente. La tercera parte, finalmente, una vez trazado este cuadro conceptual, se focalizará en el impacto de la territorialidad sobre el proceso pedagógico de orientación escolar y profesional de los alumnos.

\section{Diferenciación progresiva de las desigualdades educativas}

\section{Las principales desigualdades educativas}

Los numerosos estudios realizados en Sociología (Bourdieu et Passeron, 1964; 1970; Boudon, 1973; etc.) en la segunda parte del siglo XX y, posteriormente, en Ciencias de la Educación en el ámbito del Instituto de Investigación en Educación (IREDU), y luego en el Observatorio de la Escuela Rural $(\mathrm{OER})^{2}$ a fines del siglo $\mathrm{XX}$, entre muchos otros, han demostrado históricamente tres grandes tipos de desigualdades que atraviesan el campo educativo remitiendo a las dimensiones sociales, institucionales así como territoriales propiamente dichas, frecuentemente interpenetrándose, incrementando o atenuando sus respectivas intensidades: las desigualdades de educación, las desigualdades propiamente escolares y, dentro de éstas, las desigualdades de orientación. Es necesario diferenciarlas antes de intentar ver si, y de qué manera, los contextos territoriales (territorios y territorialidades), que están también lejos de ser objeto de un cuestionamiento previo, los impactan. Se sabe hoy, luego de los trabajos de la Sociología de la Educación, que las desigualdades sociales inducen y producen, de manera global, importantes desigualdades educativas. De manera significativa, constituyen la base de 
Espacios en Blanco - Serie indagaciones - № 30 - vol. 2 - jul./dic. 2020 (335-349)

desigualdades particulares ligadas a la escolaridad (rendimiento escolar) y a la orientación (proyectos y elecciones de centros de formación). Las desigualdades escolares se inscriben generalmente en desigualdades educativas, que aparecen efectivamente como más "amplias» que las primeras, en tanto estas últimas, las desigualdades educativas, van más allá de simples rendimientos escolares disciplinares: incluyen, por ejemplo, el aprendizaje de códigos educativos que facilitan la orientación de los alumnos y de códigos sociales que favorecen su inserción profesional.

\section{Las desigualdades de orientación}

Al profundizar en la diferenciación de estos tres tipos de desigualdades, se ha advertido, durante sucesivos estudios de campo, que las desigualdades de orientación, es decir, de construcción de la "carrera» escolar, estaban más marcadas por lo social que las desigualdades escolares propiamente dichas (Boudon, 1973; Gauthier, 2011).Los proyectos y las opciones de orientación se apoyan sobre los «niveles de aspiración» (con frecuencia traducidos torpemente como "ambiciones») y los «niveles de anticipación» (o «expectativas») de los alumnos y de sus familias que son a la vez alimentados por los «habitus» de grupo y de clase. A igual nivel escolar, por ejemplo, los alumnos que proceden de medios favorecidos elaboran proyectos de orientación más largos y generales $\mathrm{y}$, sobre todo, más selectivos, que los conducirán en términos generales a empleos más prestigiosos y mejor remunerados, que los alumnos de extracción social más «modesta» (Duru-Bellat, 2002). Caricaturizando, se podría decir que los primeros aspiran a tener éxito, imientras que los segundos esperan fracasar! Veamos ahora de qué manera y con qué intensidad las diferentes dimensiones territoriales pesan sobre la escolaridad de los alumnos.

\section{Desigualdades educativas y territorios}

Ninguna de las dimensiones de la escolarización, como hemos visto y sabemos hoy, puede liberarse completamente del contexto territorial en el cual se inscribe la acción de la escuela: forma y organización escolar, aprendizajes, desempeño, deseos, proyectos y opciones de orientación de los alumnos, didáctica y pedagogía de los docentes, etc.La escuela lleva su marca en muchos territorios. En la zona de montaña y en la zona rural aislada, por ejemplo, para hacer frente a la declinación demográfica recurrente vinculada al éxodo rural, han surgido organizaciones escolares originales: pensemos en las clases únicas en la escuela primaria, que reúnen alumnos de edades diferentes en una misma clase. El territorio es, en efecto, susceptible de influir desde el exterior sobre las diferentes dimensiones de lo escolar y no solamente sobre la organización de la escuela. Pero su influencia puede ejercerse igualmente de otro modo, como un actor educativo en ejercicio pleno (Barthes et Champollion, 2012). Puede incluso llegar a influir en la educación de manera global, sistémica, a través de los "efectos de territorio», 
como ocurre en ciertos territorios rurales montañosos franceses que han sido particularmente observados desde esta perspectiva (Champollion, 2013), como veremos más adelante, en la tercera parte.Pero ¿̇es realmente el territorio global (con todas las vertientes combinadas) lo que está en juego en la escuela o se trata más bien, e incluso ante todo, de su componente simbólico, la territorialidad, eso que, presente en las cabezas, da forma a los trayectos?

\section{Diferenciación progresiva de las dimensiones de la noción de territorio}

\section{El concepto de territorio}

El aspecto estrictamente territorial de los contextos educativos no fue completamente identificado, a nivel de sus impactos puntuales y sistémicos sobre la educación, hasta después de los años 1990 (Pesiri, 1998; Feu et Soler, 2002; Boix, 2003; Arrighi, 2004; Grelet, 2004; Caro, 2006; Champollion, 2005, 2008, 2011; entre otros). Por lo tanto, toda esta profusión conceptual atraviesa, alimentándola, a la noción multidimensional y compleja de territorio utilizada por las Ciencias Humanas y Sociales, que las investigaciones científicas -de los geógrafos en primer lugar- han destacado progresivamente y cuyas principales dimensiones constitutivas se retoman muy brevementea continuación:

- Espacial: durante mucho tiempo ha constituido el único marco, físico y geográfico en principio, de la noción emergente de territorio.

- Sociológico: pesando evidentemente sobre la escolaridad y sobre la orientación, se inscribe en el juego social de los actores del territorio que contribuye a caracterizar.

- Político e institucional: está vinculado, sobre todo, a las políticas educativas territorializadas, así como a los diferentes «efectos-maestro», «efectos-clase» $\mathrm{y}$ «efectos-establecimiento» institucionales.

- Económico: integrando los elementos constitutivos del tejido socio-económico territorial (Frémont, 1976), incluye el financiamiento de los estados nacionales y de las comunidades territoriales en particular y comprende, por lo tanto, el desarrollo de la oferta de formación y la construcción de los establecimientos.

- Simbólico: apoyado esencialmente sobre las «representaciones sociales» (Abric, 2011) del territorio en cuestión ${ }^{3}$, se refiere asimismo a los valores y las identidades que contribuye a construir.

En este artículo, nos centraremos particularmente en las siguientes tres dimensiones del territorio, en sus relaciones con lo educativo: el territorio político-institucional -para compararlo con el territorio "prescripto» del sociólogo Bernard Lahire (concepto desarrollado durante los años 1990 en su seminario de Lyon ${ }^{4}$ )-, el territorio de la acción de los actores y los habitantes - para ser comparado con el territorio "vivido» del mismo Lahire $y$, retrocediendo más atrás en el tiempo, con la «región, espacio vivido» del 
Espacios en Blanco - Serie indagaciones - № 30 - vol. 2 - jul./dic. 2020 (335-349)

geógrafo Armand Frémont (1976) -,y el territorio simbólico -para vincularlo con el territorio «soñado» de Lahire.

\section{El surgimiento de la territorialidad}

En este marco conceptual general fueron desarrollándose cada vez más, particularmente en el seno del OER y de sus socios ibéricos ${ }^{5}$, los análisis basados en el impacto de la dimensión simbólica del territorio sobre la educacióny no solamente el impacto del territorio: la «territorialidad», noción destacada por Sack (1986), Le Berre (1992) y afinada posteriormente por Gumuchian (2001), luego por Debarbieux (2008) y por Vanier (2009), en particular. La territorialidad corresponde esencialmente a la dimensión «simbólica» del territorio (Bozonnet, 1992; Ferrié, 1995; Caillouette, 2007; Aldhuy, 2008; Debarbieux, 2008; Vanier, 2009; Champollion, 2011; 2013), noción que el sociólogo Bernard Lahire había introducido, sin nombrarla de este modo, hacia fines de los años 1990 al hablar, como se mencionó anteriormente, de territorios «soñados» junto a territorios "prescriptos» y «vividos».

El territorio, visto desde esta perspectiva, corresponde de hecho a una «territorialidad activada» (Vanier, 2009). La territorialidad, dimensión territorial próxima de la «pregnancia simbólica de los espacios» (Parazelli, 2002), verdadera «representación simbólica de los lugares» (Vanier, 2009), llevada por una «conciencia colectiva» que alimenta particularmente valores e identidades(Caillouette et al, 2007), «construida y compartida» por todos sus actores (Aldhuy, 2008), está así potencialmente llena de significaciones susceptibles de alimentar identificaciones e incluso, por supuesto, en ciertos casos, contra-identificaciones. La territorialidad reenvía de hecho a un verdadero «habitus» territorial.

Territorios y territorialidades son, entonces en esta perspectiva, susceptibles de influir sobre la educación a través de múltiples y complejos vínculos. Las investigaciones que se han llevado a cabo, particularmente en la «zona de montaña» francesa, han demostrado que el factor explicativo territorial operó en gran medida luego del impacto social sobre la educación (que explicaba aproximadamente la mitad de la varianza vinculada a los contextos), así como después de los factores político-institucionales, de los efectos-maestros y de los efectos-establecimientos (que explicaban aproximadamente un tercio) (Champollion, 2013). El factor territorial explicaba un poco más de un sexto. Pero, ¿qué es un poco más, precisamente?

\section{Enfoque de las relaciones complejas territorialidades-orientación}

Breve retrospectiva histórica y desafíos sociales

Las relaciones entre orientación y territorialidades se inscriben en la problemática más amplia de la evolución de las relaciones complejas entre educación y territorios (Barthes, Champollion et Alpe, 2017). Sin embargo, la orientación escolar y profesional, como se la 
llamó en sus comienzos, no se reduce al mero adelanto de la educación: si se inscribe en este marco general, posee una historia propia y con trazos específicos.

Históricamente, hasta comienzos del siglo XX, la cuestión de la orientación no se planteaba: los hijos retomaban generalmente el oficio del padre. La problemática moderna de la orientación, surgida con los trabajos de Pierre Naville (1945), se hizo más precisa después de la Segunda Guerra Mundial en el marco de la Comisión LangevinWallon (1944-1947). La política de masificación escolar de los años 1960 fue, poco a poco, colocando a la orientación en el centro del sistema educativo: ya no se trataba solamente de adaptar al individuo al mercado de trabajo, sino también de gestionar los flujos de estudiantes, de permitir a cada uno encontrar un trabajo donde pudiera realizarse (Richit, 2014).

La orientación escolar pasó entonces, progresivamente, de un enfoque prescriptivo a un enfoque educativo que, reenviando la gestión de los flujos de estudiantes a los procedimientos administrativos, disocia lo pedagógico, es decir, la orientación, de lo administrativo, o sea de la asignación de alumnos (Caroff, 1987). Sin embargo, ¿las elecciones de orientación realizadas por los alumnos y sus padres se llevan a cabode la misma manera en todo el territorio nacional, cualesquiera sean sus especificidades, y por lo tanto, de un modo semejante en los territorios rurales montañosos y en los barrios urbanos sensibles?

\section{La orientación en los territorios rurales de montaña}

$\mathrm{Si}$ en los territorios de montaña aislados, los deseos de orientación no se presentan igual a lo que se ha comprobado en un nivel escolar equivalente en el conjunto del territorio nacional (tabla $\mathrm{n}$ - 1), sino que se mantienen muy por debajo de los promedios nacionales, la acción específica de los padres en materia de orientación limita aún más la utilización de toda la gama de alternativas de formación al orientar fuertemente la mayoría de los proyectos elaborados y las opciones efectuadas en el marco de los

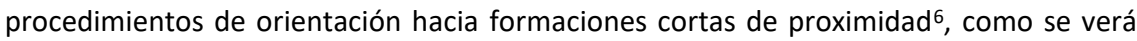
más adelante (Champollion, 2013). Los alumnos de la zona de montaña del panel OER, especialmente, en 2004 deseaban entrar en 2ㅇ GT (segundo general y tecnológico) en un 59 \%, o sea más de 10 puntos menos que a nivel nacional (Caille, 2005). 
Espacios en Blanco - Serie indagaciones - № 30 - vol. 2 - jul./dic. 2020 (335-349)

Tabla 1. Comparación de los deseos espontáneos de orientación de los alumnos de 3으 del secundario básico (collège) hacia la opción 2 a general y tecnológica en función de los diferentes tipos de ruralidad de la nomenclatura INSEE-INRA de 1996 utilizada por el OER-OET ${ }^{7}$

\begin{tabular}{|l|c|l|l|l|}
\hline Territorios & $\begin{array}{l}\text { Zona de } \\
\text { montaña }\end{array}$ & $\begin{array}{l}\text { Rural } \\
\text { aislado }\end{array}$ & $\begin{array}{l}\text { Rural } \\
\text { total }\end{array}$ & $\begin{array}{l}\text { Francia } \\
\text { Total }\end{array}$ \\
\hline $\begin{array}{l}\text { Clase } \\
\text { deseada } \\
\left(2^{\circ} \mathrm{GT}\right)\end{array}$ & $59 \%$ & $61 \%$ & $60 \%$ & $71 \%^{8}$ \\
\hline
\end{tabular}

Fuente: Encuestas OER 2003-2004 y DEPP.

Doce años más tarde, en 2016, en el marco del segundo seguimiento longitudinal OET efectuado sobre los alumnos de las mismas escuelas, se observa (tabla 2) una muy alta estabilidad de los resultados: $63 \%$ de los alumnos rurales encuestados deseaban dirigirse hacia un 20 GT contra el $61 \%$ anterior. Lo mismo sucede con los padres (54\% contra 52\%), quienes siguen constituyendo el principal freno a la aspiración de los alumnos a estudios prolongados (Champollion, 2020b, c y d).

Tabla n ․ 2. Análisis comparativo 2004/2016 de los deseos de orientación espontáneos emitidos por los alumnos de los paneles OER y OET 3 은

\begin{tabular}{lccc}
\hline Orientación & 2004 & 2016 & Diferencia 2016-2004 \\
\hline Deseo de ingresar en 2 GT - alumnos & $60 \%$ & $63 \%$ & $3 \%$ \\
Deseo de ingresar en 2 GT - padres & $52 \%$ & $54 \%$ & $2 \%$ \\
\hline
\end{tabular}

Fuente: encuestas OER-OET 2004-2016.

Esta menor aspiración a realizar estudios generales prolongados, como se ha indicado antes, se acentúa entonces de manera aún más sorprendenteentre los padres: $51 \%$, es decir 8 puntos menos que las tasas comparables de "alumnos» de la encuestaOER 2004. Esta diferenciaentre alumnos y padres, en términos de autocensura de las elecciones de orientación, requiereuna acotación: fuera de la «zona de montaña» y del área «rural aislada», sobre quienes recae el peso de la territorialidad, cuando las calificaciones escolares son altas, los padres de los alumnos, apoyados y/o impulsados por los docentes, alientan fuertemente a sus hijos a proseguir su escolaridad en dirección de los estudios generales prolongados del tipo escuela secundaria avanzada ${ }^{9}$, es decir a solicitar el ingreso en $20 \mathrm{GT}$ al finalizar el colegio.

En términos de elecciones de orientación de los alumnos de 2 o GT, la deserción de la «zona de montaña» (y también de la zona «rural aislada»), en comparación con el 
promedio de Francia, aunque sigue siendo significativa ya no es tan importante: el $31 \%$ de los alumnos de zona de montaña dicen espontáneamente que quieren ir a la 1o científica (S), presentada aquí como arquetípico de futuros estudios prolongados y generales de alumnos de secundaria avanzada ${ }^{10}$, contra el $34 \%$ en el conjunto del «espacio dominante rural» (Champollion, 2013). El «desnatado» escolar, aparentemente, ya ha hecho su trabajo. Sin embargo, la dirección global de las tendencias observadas anteriormente se mantiene ampliamente: cuanto más nos acercamos al aislamiento, menos son demandados los estudios largos y generales (tabla no 3).

Tabla $n^{\circ} 3$. Alumnos en 2o GT (2005): comparación de los porcentajes de los deseos espontáneos hacia las diversas series de bachillerato entre los diferentes tipos de territorio rural-montañoso.

\begin{tabular}{|l|c|}
\hline Territorios & Referencias de orientación espontáneas al1 ${ }^{\circ}$ S \\
\hline Zona de montaña & $31 \%$ \\
\hline Rural aislado & $32 \%$ \\
\hline Rural total & $34 \%$ \\
\hline Francia total & $36 \%$ \\
\hline
\end{tabular}

Fuente: encuesta OER 2004-2005 y RERS 2006.

\section{La adaptación de la escuela a los contextos locales}

Recordemos -para mantenernos solamente en el contexto de montaña- que la primera ley de montaña de 1985, en sus artículos 10 y 11, estipulaba que el sistema educativo tenía que "adaptarse" al contexto montañoso ${ }^{11}$. Sin embargo, si nos remitimos a los proyectos de establecimientos (aquí, colegios secundarios) frecuentados por los alumnos del panel OER, se puede comprobar que, en sus principales ejes, la orientación es priorizada solo en el 39 \% de los casos, muy por debajo del éxito escolar; es decir, que lo es en el $82 \%$ de los casos, mientras que los alumnos del panel tanto de escuela primaria como de secundaria básica, obtienen resultados escolares ligeramente superiores al promedio de sus congéneres (Champollion, 2005). La educación de orientación, destinada a ayudar a los alumnos a acceder, sin censura o autocensura, a formaciones largas y generales que les pudieran interesar, no está entonces realmente integrada en la política del establecimiento puesto que, en la mayoría de los casos, la temática de la orientación no constituye realmente uno de sus ejes prioritarios. 
Espacios en Blanco - Serie indagaciones - № 30 - vol. 2 - jul./dic. 2020 (335-349)

Tabla $n^{\circ} 4$. Principales ejes de los proyectos de establecimiento de los colegios secundarios de la «base» general rural ${ }^{12}$

\begin{tabular}{|l|c|}
\hline $\begin{array}{l}\text { Áreas prioritarias de los proyectos del } \\
\text { establecimiento }\end{array}$ & \% de escuelas \\
\hline Exitoescolar & $82 \%$ \\
\hline Salud/Ciudadanía & $51 \%$ \\
\hline Apertura & $43 \%$ \\
\hline Orientación & $39 \%$ \\
\hline
\end{tabular}

Fuente: encuesta 2003-2004 «establecimientos» OER.

¿Las diferentes especificidades de la escuela rural de montaña que, como se ha visto, en materia de orientación, corresponden a otras tantas desigualdades, remiten a causalidades puntuales más bien yuxtapuestasy, por consiguiente, sin muchos vínculos entre ellas ?, ¿o bien pueden formar parte de un conjunto causal más global?

\section{¿«Efectos territoriales» sobre la educación?}

¿Cómo explicar esta diferencia entre lo rural en general y los territorios rurales montañosos aislados de los paneles OER-OET por un lado, y por otro, el hecho de que toda Francia, en términos de los comportamientos de orientación de los alumnos, no presenten diferencias significativas en materia de resultados escolares? Muchos factores influyen conjuntamente sobre estas prácticas de orientación aparentemente paradojales. Repulsión frente a la movilidad, dificultad para proyectarse en el futuro lejano, alejamiento y/o carencia de ofertas de formación, etc., se combinan, en primer lugar, para impedir que los alumnos rurales de montaña "capitalicen» en términos de orientación sus buenos resultados escolares. Otro tipo de explicación, vinculada más directamente al "capital humano», es decir a la formación de los docentes, podría también intervenir aquí: la casi-ausencia de formación específica en los planes de formación de las Escuelas Superiores del Profesorado y de la Educación (ESPE) ${ }^{13}$, que reemplazaron, en 2013, a los Institutos Universitarios de Formación de Maestros (IUFM), en las características particulares de los procesos de orientación en el trabajo en territorios rurales de montaña. Finalmente, a nivel institucional, la configuración escolar, la organización del tejido educativo, el alejamiento de la oferta de formación, la cantidad de alumnos en las clases, las estrategias de ruptura del aislamiento, etc., que reenvían también ellos a otros tantos aspectos de la escolarización rural de montaña, y juegan evidentemente un rol en el proceso de orientación...

Un «efecto de territorio» sobre la educación, inicialmente observado al final de la escuela secundaria básica (Champollion, 2005) ha sido luego confirmado al inicio de la secundaria de ciclo superior ${ }^{14}$, a nivel de la 2 a GT (Champollion, 2008). En el marco de 
varios análisis factoriales exploratorios de correspondencias, realizados sobre los datos de 3 o yluego de 2 - GT recolectados por el OER-OET, dos tipos de explicación han sido sucesiva y claramente puestos en evidencia: el "peso del anclaje territorial» y la "capacidad de proyectarse en un futuro lejano». Estas dos explicaciones se combinan técnicamente para constituir, juntas, el efecto de territorio (Champollion, 2005, 2008, $2013)^{15}$. En los territorios aislados rurales y montañosos que han sido estudiados, en efecto, el peso conjunto de lo «local» y de lo «inmediato», correspondiente a aproximadamente el $50 \%$ de la variación territorial explicada, reenvía a un factor explicativo global, a la vez compuesto y difuso, el efecto de territorio que permite comprender mejor los fenómenos paradójicos fundamentales identificados. Estos efectos de territorio territorializan los proyectos de orientación escolar elaborados por el conjunto de actores del proceso pedagógico (estudiantes del ciclo básico y superior de la escuela secundaria, padres, docentes y personal educativo): al dirigirse principalmente en dirección a una mayor modestia y a una mayor cercanía en los proyectos concebidos, reducen de hecho la amplitud de opciones de orientación disponibles para los alumnos de la zona montañosa.

Vinculado particularmente con la territorialidad de montaña, este efecto de territorio, o más bien este efecto de territorialidad, explica lo que global y paradojalmente se ha constatado en la "zona de montaña» y en la zona "rural aislada»: los buenos resultados escolares obtenidos por los alumnos del panel OER en la primaria -que son confirmados en la secundaria- no producen en materia de orientación, recordémoslo, los mismos efectos que en cualquier otro lugar de Francia. De un modo sorprendente, debido en particular a una sobre determinación del anclaje territorial, generadora de una menor movilidad potencial $y$ de una capacidad menor de "proyectarse» en el futuro ${ }^{16}$, los alumnos y sobre todo los padres de los alumnos de los territorios rurales montañosos -en mayor o menor medida según el tipo de territorio montañoso, más en la meseta de Ardeche, menos en el alto valle del Ubaye (Champollion \& Legardez, 2010), más o menos según el género de los alumnos, paradójicamente más para los muchachos que para las chicas (May-Carle, 2012; Meunier, 2015)- en su mayoría no utilizan tanto como los demás la gama completa de opciones de orientación disponibles en el final de 3 y de 2ㅇ GT (Champollion, 2005, 2008, 2013). Este fenómeno, que comienza "soterradamente» a partir de los sueños y las proyecciones escolares de los alumnos y los padres a fines de la primaria, se desarrolla tanto en los alumnos como, en particular, en los padres $y$, en una menor medida, en los docentes. Este impacto territorial puede llegar hasta contribuir a «encerrarlos» parcialmente en su entorno local por la vía de un «formateo» cultural (que es lo que ocurre mayoritariamente) o bien, al contrario, liberarlos parcial y progresivamente mediante una toma de conciencia de la intensidad de su anclaje territorial a través de un trabajo pedagógico ${ }^{17}$. 
Más allá de este efecto de territorio singular, que se ha manifestado en la "zona de montaña» francesa, ¿no podrían existir varios tipos de efecto de territorio, característicos de ciertos territorios y no de otros? ¿No habría entonces, finalmente, múltiples efectos de territorio, específicos, por ejemplo, de áreas educativas prioritarias, más allá de las políticas educativas desarrolladas en estos territorios desfavorecidos? ¿O bien, específicos de centros urbanos «burgueses»? ¿O bien, específicos de zonas premontañosas? ¿O bien, incluso, específicos de zonas costeras? ¿O bien, específicos de territorios isleños?En un nivel más teórico, notamos que un efecto de territorio sobre el sistema escolar, tal como hoy puede caracterizarseprovisoriamente este concepto original surgido de encuestas de campo a partir de sus dos primeras comprobaciones en territorios rurales montañosos, se manifiesta aparentemente de manera global y sistémica.Abarca el conjunto de las diferentes variables educativas dependientes, o al menos, una subparte significativa de éstas, aparte, por supuesto, de las variables de pertenencia o ambientales. Amplifica o contradice fenómenos complejos socialmente esperados, o incluso, determina nuevos (Champollion, 2013).

\section{Conclusión}

Durante el desarrollo de este artículo, hemos podido percibir que el contexto territorial, en la literatura científica era reducido,muy frecuentemente, a sus dimensiones espaciales (tomadas en el sentido de caracteres territoriales físicos, es decir, relacionados con el clima, la superficie, la altitud, etc.) y las instalaciones locales del territorio (distancia o proximidad y diversificación o concentración de la oferta educativa, por ejemplo), institucionales (políticas educativas, por ejemplo, o bien «efectos establecimiento») y financieros (contribuciones dependientes del Estado y de las colectividades territoriales, particularmente). Se olvida demasiado rápido que las dimensiones simbólicas o territorialidades (vinculadas entre otras cosas, como hemos visto, a representaciones, valores e identidades) pesan claramente, de una manera muy significativa,en los proyectos y las elecciones de los alumnos y sus padres,en particular en materia de orientación, contribuyendo -junto a otros factores contextuales, por supuesto- en convertir en desiguales sus trayectorias, en mayor o menor medida según los tipos de territorio(Champollion, 2019a; Bellarbre \& Champollion, 2020).

Sin embargo, sería inexacto creer que los impactos de las diferentes dimensiones territoriales constituyen, esencial y obligatoriamente, invariantes contra las cuales no se podría hacer absolutamente nada. Ciertamente, existen muchos determinantes territoriales, así como existen determinantes sociales de intensidad más fuerte, pero no hay en la materia, sin embargo, un determinismo automático lindante con el fatalismo. Tratándose de la orientación, es seguramente posible, por ejemplo, permitiendo a los alumnos rurales y montañeses combatir su propia auto-censura ayudándolos a tomar conciencia de que una parte de sus elecciones personales no reflejan su libertad 
individual, sino el peso de la territorialidad. La debilidad de la formación profesional de los docentes en las particularidades de los territorios -y por lo tanto, de los públicos en los que impactan- y de la pedagogía de orientación de los docentes, hace que la enseñanza que brindan sea necesariaseguramente para esto.No olvidemos que, de hecho, el compartir, alumnos y padres una misma territorialidad -quienes nacieron allí-, y los docentes que lo habrían aprendido -en la formación inicial o continua o en el terreno-es probable que elimine muchas de las dificultades encontradas en este campo(Champollion \& Rothenburger, 2018).

Finalmente -esta es una línea de investigación que parece muy prometedora- ahora es posible pensar que los territorios son todavía y muy frecuentemente aprehendidos a través de categorizaciones demasiado simplistas, rígidas y globales (lo rural, lo urbano, etc.), aunque comienza a surgir actualmente una cierta diferenciación territorial, ya sea afinando la tipología disponible de los espacios y de los territorios como lo ha hecho en muchas oportunidades el Instituto Nacional Estadística y Estudios Económicos (INSEE), ya sea distinguiendo en el seno mismo de la noción de territorio muchas dimensiones, como lo han hecho los sociólogos (Lahire) y muchos geógrafos (de Le Berre a Vanier), ya sea incluso combinando varios criterios (densidad de población, migraciones diarias domicilio-trabajo, polarización económica, etc.) como lo hace actualmente la Dirección de Evaluación, Prospectiva y Desempeño (DEPP).

Mientras que, para atenerse solo a los territorios rurales-montañosos investigados en los estudios que subyacen a este artículo, hay por ejemplo tantos territorios de montaña como macizos - ila meseta de Ardèche no es ni Bauges ni el alto Ubaye!incluso si entre ellos existen obviamente regularidades. El desarrollo de los análisis pertinentes en materia de interrelaciones educación-territorio tiene seguramente, entre otras cosas, jeste costo!

\section{Notas}

\footnotetext{
${ }^{1}$ Escuelas, «collèges» (secundario 1 ) y «lycées» (secundario 2 ).

2 Se convirtió en 2009 en Observatorio de Educación y Territorio (OET) debido a la ampliación de su campo de investigación a todos los tipos de territorio (incluidos los urbanos): http://observatoire-education-territoires.com.

${ }^{3}$ Una parte no despreciable de esta dimensión simbólica, que va más allá del simple registro sociocognitivo de la «representación», corresponde probablemente también a una «interiorización» (Merton, 1949) dependiente del «inconsciente colectivo» (Jung, 1988).

${ }^{4}$ Grupo de investigación sobre la socialización.

${ }^{5}$ En el marco del Proyecto EDUC13460 2009-2012 que reunió a las universidades de Barcelona, Granada, Lisboa y Zaragoza.

${ }^{6}$ No se trata aquí, evidentemente, de oponer las formaciones prolongadas y generales, que serían más «nobles», a las formaciones cortas y profesionales, jerarquizando las vías de formación: la desigualdad en cuestión proviene únicamente de la posible autorestricción del abanico de alternativas de formación hacia las más modestas y cercanas.

${ }^{7}$ Champsaur (1998).

${ }^{8}$ Panel DEPP 1995 que es el más cercano del panel OER en materia de deseos espontáneos de orientación (Caille, 2005).

${ }^{9}$ En el original: lycée (NdeT).

${ }^{10}$ En el original: lycéens (NdeT).

${ }^{11}$ Lo que fue confirmado por la segunda ley de «montaña» de 2005.
} 


\begin{abstract}
12 Recordemos entonces que al nivel de la zona "rural aislada» especialmente, las características se aproximan mucho a las de la «zona de montaña », de la media en particular (Mériaudeau, 1980).

${ }^{13}$ Los programas de formación de los nuevos Institutos Nacionales superiores del profesorado y de la educación (INSPE) que reemplazaron a los ESPE aún no están disponibles.

${ }^{14}$ Se ha traducido "collège" y "lycée" como "escuela secundaria básica" y "escuela secundaria de ciclo superior" por entender que son los términos que mejor corresponden a la estructura del sistema educativo francés en comparación con el argentino.(NdeT)

${ }^{15}$ Para obtener más detalles técnicos sobre la definición de este concepto, remitirse a las páginas 103-121 de la obra de Pierre Champollion titulada «Les inégalités d'éducation et d'orientation d'origine territoriale» indicada en la bibliografía.

${ }^{16}$ Hemos encontrado esta misma característica de dificultad de proyectarse en el futuro identificada en «zona de montaña» en muchos territorios: en baldíos industriales (à Seraing, por ejemplo, cerca de Liège, en Bélgica), en territorios urbanos «devastados» por la recesión económica, por ejemplo, o bien en zonas peri-urbanas más bien desfavorecidas como el Blayais próximo a Bordeaux, o incluso zonas rurales no montañosas relativamente aisladas como Guéret, Vesoul, Foix, etc.

17 A título de evidencia a veces olvidada, resulta útil reiterar aquí, de paso, que la medida por parte de los investigadores de la influencia del factor territorial no debería más que otros, conducir ipso facto a los actores de la orientación a adoptar comportamientos «deterministas». Al contrario, hacer tomar conciencia a todos los actores de la orientación de los impactos de los «determinantes» estadísticos, territoriales en este caso, de la orientación, no puede sino ayudarlos, si no a liberarse de esos impactos al menos a aminorarlos. Porque es al ejercicio progresivo de una libertad supervisada, restringida a veces, a lo que deberían ser invitados los alumnos de la secundaria.
\end{abstract}

\title{
Bibliografía
}

Abric, J.C. (dir.) (2011). Pratiques sociales et représentations. Paris: PUF.

Aldhuy, J. (2008). “Au-delà du territoire, la territorialité?”. EnGéodoc, n 55, pp. 35-42.

Arrighi, J.J. (2004)."Les Jeunes dans l'espace rural: une entrée précoce sur le marché du travail ou une migration probable". En Formation-Emploi, n 87, pp.68-78.

Barthes, A. \& Champollion, P. (2012). "Éducation au développement durable et territoires ruraux. Problématique, projet de territoire et réduction fonctionnelle de l'enseignement. Education relative à l'environnement". En Regards. Recherches. Réflexions, n 10, pp. 36-51.

Barthes, A., Champollion, P. \&Alpe, Y. (dir.) (2017). Permanences et évolutions des relations complexes entre éducations et territoires. Londres: ISTE.

Bellarbre, E. \& Champollion, P. (2020). "Evolutions récentes des représentations territoriales et scolaires des élèves de collège ruraux-montagnards susceptibles d'intervenir dans la construction des inégalités d'orientation d'origine territorial”. In Régis Malet (dir.) Éducation, mondialisation et citoyenneté. Enjeux démocratiques et pratiques culturelles. Bern: Peter Lang éditions. Sous pressé.

Boix, R. (2003). "Las zonas Escolares Rurales en Cataluna". En Organizacion y gestion dos centros educativos. Barcelona: Editorial Praxis.

Boudon, R. (1973). L'inégalité des chances. La mobilité sociale dans les sociétés industrielles. Paris: A. Colin.

Bourdieu, P. \& Passeron, J.C. (1970). La reproduction. Éléments pour une théorie du système d'enseignement. Paris: Editions de Minuit.

Bourdieu, P. \& Passeron, J.C. (1964). Les héritiers. Paris: Editions de Minuit.

Bozonnet, J.P. (1992). Des monts et des mythes. L'imaginaire social de la montagne. Grenoble: PUG.

Caille, J.P. (2005). "Le vécu des phases d'orientation en fin de troisième et de seconde". En Éducation \& Formations, 72.

Caillouette, J. et al. (2007). "Territorialité, action publique et développement des communautés". En Économie et solidarités, 38, 1, pp. 9-23. 
Caro, P. (2006). "La dimension partiale des systèmes emploi-formation". En L'Espace géographique, $n^{\circ} 3$, pp. 223-240.

Caroff, A. (1987). L'organisation de l'orientation des jeunes en France. Paris: EAP.

Champollion, P. (dir.) (2020). Territorialisation de l'éducation. Tendance ou nécessité. Londres: ISTE.

Champollion, P. (2019). Inégalités d'orientation et territorialité: l'exemple de l'école rural e montagnarde. Contribution au rapport CNESCO « Justice à l'école et territoires ». Paris: Cnesco. 43p. (disponible sur le site du Cnesco: http://www.cnesco.fr).

Champollion, P. (2020b). "Représentations territoriales et scolaires rurales: atténuation de leurs spécificités et maintien de poches de résistance". In Champollion P. (dir) Territorialisation de l'éducation. Tendance ou nécessité. Londres: ISTE.

Champollion, P. (2020c). "Rapprochement des représentations rurales et urbaines en raison de la pénétration d'internet et des réseaux o cciaux?" In Champollion P. (dir) Territorialisation de l'éducation. Tendance ou nécessité. Londres: ISTE.

Champollion, P. (2020d). "Etat de la question "éducation et territoire": analyse à partir de territoires ruraux et montagnards". In Danic, I., David, O. \& al. Les espaces de construction des inégalités éducatives. Rennes: PUR.

Champollion, P. (2015). "Education and territory: conceptual framework". In Boix, R., Champollion, P. \& Duarte, A. (coord.) Territorial Specificities of Teaching and Learning, Sisyphus - Journal of Education. Recuperado de: https://doi.org/10.25749/sis.7882.

Champollion, P. (2013). Les inégalités d'éducation et d'orientation d'origine territoriale. Collection Crise et anthropologie de la relation. Paris: L'Harmattan.

Champollion, P. (2011). École et territoire: de l'impact du territoire à l'effet de territoire.(Habilitation à diriger des recherches) Université Paul Valéry, France.

Champollion, P. (2008). "La Territorialisation du processus d'orientation en milieux ruraux et montagnards: de l'impact du territoire à l'effet de territoire". En Éducation et formations, $\mathrm{n}^{\circ} 77$, L'orientation, pp. 43-53.

Champollion, P. (2005). Impact de la scolarisation en "zone de montagne» sur la réussite scolaire et I'orientation (Thèse de doctorat). Université de Provence (Aix-Marseille1), France.

Champollion, P. \& Rothenburger, C. (2018). "De la didactique du territoire au territoire apprenant". In Diversité, $n^{\circ} 191$, pp.54-63.

Champollion, P. \& Legardez, A. (2010). "Scolarisation en micro-territoires ruraux".En Alpe, Champollion, P. \& Poirey, J.-L. (coord.).L'Enseignement scolaire en milieu rural et Montagnard, Tome 5, Après le collège. Besançon: Presses Universitaires Franc Comtoises, pp. 91-96.

Champsaur, P. (dir.) (1998). Les Campagnes et leurs villes. INRA-INSEE.

Debarbieux, B. (2008). “Construits identitaires et imaginaires de la territorialité: variations autour de la figure du montagnard". En Annales géographiques, 660-661, pp. 90-115.

Duru-Bellat, M. (2002). Les Inégalités sociales à l'école: genèse e tmythes. Paris: PUF.

Ferrier, J.P. (1995). Le contrat géographique ou l'habitation durable des territoires. Lausanne: Payot.

Feu, J. \& Soler, J. (2002)."Mésenllà de l'escola rural: cap a un model integral i integrador de l'educació en el territorio". En Temps d'Educació, n²6, pp. 133-156.

Frémont, A. (1976). La région, espace vécu. Paris: PUF.

Gauthier, J. (2011). En quoi l'école est-elle inégalitaire? Skholé, nº 12.

Grelet, Y. (2004). "La reproduction sociales 'inscrit dans le territoire”.En Formation-Emploi, n 87. Paris: La Documentation Française. 
Espacios en Blanco - Serie indagaciones - № 30 - vol. 2 - jul./dic. 2020 (335-349)

Gumuchian, H. (2001). "École, territoire et développement durable". En Alpe, Champollion, P. \& Poirey, J.-L. (coord.). L'Enseignement scolaire en milieu rural et montagnard, Tome 1, Espaces ruraux et réussites scolaires, Besançon: Presses universitaires franc-comtoises.

Jung, C. G. (1988). Essai d'exploration de l'inconscient. Paris: Gallimard.

Le Berre, M. (1992). "Territoires". In Bailly, A., Ferras, R. \& Pumain, D. (dir.) Encyclopédie de géographie. Paris: Economica.

May-Carle, T. (2012). Les effets des contextes territoriaux ruraux sur les trajectoires scolaires des garçons et des filles: l'exemple du rural isolé et du rural sous faible influence urbaine (Thèse de doctorat). Aix-Marseille université, France.

Mériaudeau, R. (1980)."L'enfant, l'école et la montagne. In Gumuchian, H. \&Mériaudeau, R., L’Enfant montagnard...son avenir?" En Revue de Géographie Alpine, n hors-série, pp. 69-125.

Merton, R. K. (1949). Social Theory and Social Structure. New York: The free press.

Meunier, B. (2015). Contextes territoriaux et trajectoires scolaires: le cas des filles issues des milieux ruraux (Thèse de doctorat). Aix-Marseille université, France.

Naville, P. (1945). Théorie de l'orientation professionnelle. Paris: Gallimard.

Parazelli, M. (2002). La rue attractive. Parcours et pratiques identitaires des jeunes de la rue. Québec:Presses de l'Université du Québec.

Pesiri, A. (coord.) (1998). Serino ExtraMuros: L'Orientamento nella scuola de Il'autonomia. Napoli: Istituto Grafico Editoriale italiano.

Richit, N. (2014). "L'éducation à l'orientation dans le cadre d'une enseignement d'exploration en seconde". En Recherches en Didactiques, 18, pp.101-118

Sack, R.D. (1986). Human territoriality. Cambridge: University Press.

Vanier, M. (2009). Territoires, territorialité, territorialisation. Controverses et perspectives. Rennes: PUR. 